Comment. Math. Helv. 78 (2003) 648-662 0010-2571/03/030648-15

DOI $10.1007 / \mathrm{s} 00014-003-0770-0$ (c) 2003 Birkhäuser Verlag, Basel

Commentarii Mathematici Helvetici

\title{
Presentations of the first homotopy groups of the unitary groups
}

Thomas Püttmann and A. Rigas

\begin{abstract}
We describe explicit presentations of all stable and the first nonstable homotopy groups of the unitary groups. In particular, for each $n \geq 2$ we supply $n$ homotopic maps that each represent the $(n-1)$ !-th power of a suitable generator of $\pi_{2 n} \mathrm{SU}(n) \approx \mathbb{Z}_{n !}$. The product of these $n$ commuting maps is the constant map to the identity matrix.
\end{abstract}

Mathematics Subject Classification (2000). Primary 57T20.

Keywords. Homotopy groups, compact Lie groups, equivariant maps.

\section{Introduction}

The homotopy groups of compact Lie groups have been of continuous interest since the discovery of homotopy groups at around 1935. There is now a tremendous amount of computational tools available and many groups have been determined. On the other hand, intellectually and practically satisfying presentations of these groups are only known in comparatively few cases. Our goal in this paper is to describe such presentations for the first homotopy groups of the unitary groups.

For the stable groups we mainly, but not entirely, review some known results and procedures in an easily accessible and most explicit way. Particular emphasis is given to the last stable groups $\pi_{2 n-1} \mathrm{U}(n)$. A highlight of this part is a strikingly simple formula for a minimal embedding of $\mathbb{S}^{5}$ into $\mathrm{SU}(3)$ that represents a generator of $\pi_{5} \mathrm{SU}(3)$ and has a natural interpretation in terms of the complex cross product.

The main achievement of the paper concerns the first nonstable homotopy groups $\pi_{2 n} \mathrm{SU}(n) \approx \mathbb{Z}_{n !}$. These groups played an important role in the first proofs of the fact that the only parallelizable spheres are $\mathbb{S}^{1}, \mathbb{S}^{3}$, and $\mathbb{S}^{7}$. We figure in an elementary and explicit way how a suitable generator of $\pi_{2 n} \mathrm{SU}(n)$ becomes null-homotopic in the $n$ !-th power. Namely, we supply $n$ homotopic maps that

The joint work of the authors was supported by CNPq and the International Bureau of the $\mathrm{BMBF}$ in the scope of the former $\mathrm{CNPq} / \mathrm{GMD}$-agreement. 
each represent the $(n-1)$ !-th power of the generator. The product of these $n$ commuting maps is the constant map to the identity matrix. The generators of $\pi_{2 n} \mathrm{SU}(n)$ are then used to produce similar presentations of the homotopy groups $\pi_{2 n} \mathrm{SU}(n-1)$ with even $n$.

In the final section we obtain presentations of certain stable homotopy groups of the symplectic groups and a structure theorem for certain nonstable homotopy groups of the symmetric spaces $\mathrm{SU}(n) / \mathrm{SO}(n)$.

Throughout this paper we use the well-known fact that the homotopy group $\pi_{k}(G)$ of a compact connected Lie group $G$ is isomorphic to the group of free homotopy classes of maps $\mathbb{S}^{k} \rightarrow G$. Here, the product between two free homotopy classes is given by multiplying the representing maps value by value with the product of $G$. We also often use the elementary fact that the inclusions $\operatorname{SU}(n) \rightarrow$ $\mathrm{U}(n)$ induce isomorphisms between $\pi_{r} \mathrm{SU}(n)$ and $\pi_{r} \mathrm{U}(n)$ for $r \geq 2$.

\section{The stable homotopy groups of the unitary groups}

\subsection{Bott periodicity}

It has been known since around 1940 that the inclusion of $\mathrm{U}(n)$ into $\mathrm{U}(n+1)$ induces an isomorphism between the homotopy groups $\pi_{r} \mathrm{U}(n)$ and $\pi_{r} \mathrm{U}(n+1)$ if $r<2 n$. The homotopy groups in this range are called stable. Their simple structure became visible at the end of the 50's by Bott's famous periodicity theorem [3]: The stable groups $\pi_{r} \mathrm{U}$ are trivial if $r$ is even and isomorphic to $\mathbb{Z}$ if $r$ is odd. In fact, Bott constructed isomorphisms

$$
\pi_{r} \mathrm{U}(n) \rightarrow \pi_{r+2} \mathrm{SU}(2 n)
$$

for $r<2 n$ and thus all stable groups are determined by $\pi_{1} \mathrm{U}(1) \approx \mathbb{Z}$ and the trivial group $\pi_{2} \mathrm{U}(2)$. The periodicity isomorphisms can be given in the following explicit way: One assigns to a map $\theta: \mathbb{S}^{r} \rightarrow \mathrm{U}(n)$ the map $\mathcal{B}(\theta): \mathbb{S}^{r+2} \rightarrow \mathrm{SU}(2 n)$ defined on the unit sphere in $\mathbb{C} \times \mathbb{R}^{r+1}$ by

$$
\mathcal{B}(\theta)\left(\begin{array}{c}
w \\
x
\end{array}\right)=\left(\begin{array}{cc}
\mathbb{1} & 0 \\
0 & \theta(\hat{x})
\end{array}\right)\left(\begin{array}{cc}
w \mathbb{1} & -|x| \mathbb{1} \\
|x| \mathbb{1} & \bar{w} \mathbb{1}
\end{array}\right)\left(\begin{array}{cc}
\mathbb{1} & 0 \\
0 & 0 \\
\hline \theta(\hat{x})^{t}
\end{array}\right)=\left(\begin{array}{cc}
w \mathbb{1} & -|x| \overline{\theta(\hat{x})}^{t} \\
|x| \theta(\hat{x}) & \bar{w} \mathbb{1}
\end{array}\right) .
$$

Here, $\hat{x}$ stands as an abbreviation for the unit vector $\frac{x}{|x|} \in \mathbb{S}^{r}$ and $\mathbb{1}$ denotes the $n \times n$ identity matrix. This assignment $\mathcal{B}$ provides the periodicity isomorphism. We refer to [7], [8], and [10] for (essentially) this form of $\mathcal{B}$. In [7], [8] it is deduced by the relation to Hurwitz-Radon matrices with the help of $K$-theory. In [10], Bott's original arguments [3] are turned into an explicit formula. 


\subsection{Totally geodesic presentations}

Iterating the periodicity isomorphism $\mathcal{B}$ above starting with the parametrization

$$
\zeta_{1}: \mathbb{S}^{1} \rightarrow \mathrm{U}(1), \quad z \mapsto z
$$

provides embeddings $\zeta_{k}: \mathbb{S}^{2 k-1} \rightarrow \mathrm{U}\left(2^{k-1}\right)$ that represent generators of the groups $\pi_{2 k-1} \mathrm{U}\left(2^{k-1}\right)$ and take values in $\mathrm{SU}\left(2^{k-1}\right)$ if $k \geq 2$. For example,

$$
\zeta_{2}: \mathbb{S}^{3} \rightarrow \mathrm{SU}(2), \quad\left(\begin{array}{c}
w \\
z
\end{array}\right) \mapsto\left(\begin{array}{cc}
w & -\bar{z} \\
z & \bar{w}
\end{array}\right)
$$

is the standard parametrization of $\mathrm{SU}(2)$ and

$$
\zeta_{3}: \mathbb{S}^{5} \rightarrow \mathrm{SU}(4), \quad\left(\begin{array}{c}
z_{1} \\
z_{2} \\
z_{3}
\end{array}\right) \mapsto\left(\begin{array}{rrrr}
z_{1} & 0 & -\bar{z}_{2} & -\bar{z}_{3} \\
0 & z_{1} & z_{3} & -z_{2} \\
z_{2} & -\bar{z}_{3} & \bar{z}_{1} & 0 \\
z_{3} & \bar{z}_{2} & 0 & \bar{z}_{1}
\end{array}\right)
$$

The embeddings $\zeta_{k}$ are totally geodesic and $\mathbb{R}$-linear in the sense that they extend to $\mathbb{R}$-linear maps from $\mathbb{R}^{2 k}$ to the space of complex $2^{k-1} \times 2^{k-1}$ matrices. By placing several copies of $\zeta_{k}$ or its inverse along the diagonal in a sufficiently large square matrix one can realize all elements of the homotopy group $\pi_{2 k-1} \mathrm{U}$ by totally geodesic, $\mathbb{R}$-linear embeddings. For all these and additional facts we refer to [7], [8], and [10].

We have just seen that the homotopy groups $\pi_{2 k-1} \mathrm{U}(n)$ admit very simple presentations if $k$ is very small compared to $n$. The question we are now going to answer is how one can obtain presentations of the last stable groups $\pi_{2 n-1} \mathrm{U}(n)$ in the sequence $\pi_{r} \mathrm{U}(n)$ with fixed $n$.

\subsection{A deformation}

Consider the subset of $\mathrm{SU}(n+1)$ that consists of matrices whose lower right entry vanishes. There is the following map from this subset to the group $\mathrm{SU}(n)$ :

$$
\left(\begin{array}{cc}
A & b \\
\bar{c}^{t} & 0
\end{array}\right) \mapsto A-b \bar{c}^{t} .
$$

Here $A$ is an $n \times n$-matrix and $b, c$ are unit vectors in $\mathbb{C}^{n}$. The map above can be obtained by the following deformation in $\mathrm{SU}(n+1)$ :

$$
\left(\begin{array}{cc}
A-b \bar{c}^{t} \sin t & b \cos t \\
\bar{c}^{t} \cos t & \sin t
\end{array}\right)
$$

For $t=0$ we get the initial matrix above and for $t=\frac{\pi}{2}$ we obtain the target matrix embedded in the upper left $n \times n$-block of $\mathrm{SU}(n+1)$. This deformation exists analogously in $\mathrm{SO}(n+1)$ and $\mathrm{Sp}(n+1)$. 


\subsection{Factorization of the periodicity isomorphism}

Because of stability the original periodicity isomorphism $\mathcal{B}$ admits the following factorization:

$$
\pi_{r} \mathrm{U}(n) \stackrel{\mathcal{B}^{\prime}}{\longrightarrow} \pi_{r+2} \mathrm{SU}(n+1) \rightarrow \pi_{r+2} \mathrm{SU}(2 n) .
$$

In order to obtain this factorization explicitly, we review essentially an algorithm of Lundell [16]. There are, however, modifications in the details and we substitute some of his arguments by the simple explicit deformation above. The algorithm itself is very short: In a first step one deforms the map $\mathcal{B}(\theta)$ with values in $\mathrm{SU}(2 n)$ by multiplying the matrix

$$
\left(\begin{array}{lcc}
11 & 0 & 0 \\
0 & \cos t & -\sin t \\
0 & \sin t & \cos t
\end{array}\right)
$$

from the left. For $t=\frac{\pi}{2}$ the lower two rows in each value of $\mathcal{B}(\theta)$ are exchanged (one changes the sign). Hence, the lower right entry of the resulting matrix valued map vanishes. In a second step one now applies the deformation to $\mathrm{SU}(2 n-1)$ described above. It is not complicated to check that these two deformation steps can be iterated until the map takes values in $\mathrm{SU}(n+1)$. This yields the factorized isomorphism $\mathcal{B}^{\prime}: \pi_{r} \mathrm{U}(n) \rightarrow \pi_{r+2} \mathrm{SU}(n+1)$ for $r \leq 2 n-1$. Note that for arbitrary $r$ the map $\mathcal{B}^{\prime}$ is still a homomorphism. The effect of this homomorphism on the nonstable homotopy groups of the unitary groups is studied in [16]. For example, it is shown that a generator of $\pi_{2 n} \mathrm{SU}(n) \approx \mathbb{Z}_{n}$ ! is mapped to $(n+1)$ times a generator of $\pi_{2 n+2} \mathrm{SU}(n+2)$.

\subsection{The last stable groups $\pi_{2 n-1} \mathrm{U}(n)$}

Iterating the factorized version of the periodicity isomorphism starting with the map $\eta_{1}=\zeta_{1}$ above we obtain maps $\eta_{n}$ that represent generators of the last stable groups $\pi_{2 n-1} \mathrm{U}(n)$ and take values in $\mathrm{SU}(n)$ for $n \geq 2$. Note that $\eta_{2}=\zeta_{2}$ still is the standard parametrization of $\mathrm{SU}(2)$. In the case $n=3$ one applies the deformation above to the generator $\zeta_{3}$ of $\pi_{5} \mathrm{SU}(4)$. This yields the map

$$
\eta_{3}: \mathbb{S}^{5} \rightarrow \mathrm{SU}(3), \quad\left(\begin{array}{c}
z_{1} \\
z_{2} \\
z_{3}
\end{array}\right) \mapsto\left(\begin{array}{ccc}
z_{1}+\bar{z}_{3} z_{2} & -\bar{z}_{3}^{2} & -\bar{z}_{2}+\bar{z}_{3} \bar{z}_{1} \\
z_{2}^{2} & z_{1}-z_{2} \bar{z}_{3} & z_{3}+z_{2} \bar{z}_{1} \\
-z_{3}+\bar{z}_{1} z_{2} & -\bar{z}_{2}-\bar{z}_{1} \bar{z}_{3} & \bar{z}_{1}^{2}
\end{array}\right) .
$$

A map of this form was obtained by Chaves and Rigas [5] with a related but slightly more complicated approach. With a few transformations we simplify the formula in a way that a striking relation to the complex cross product appears and its equivariance properties are revealed. In fact, after multiplying $\eta_{3}$ by $\left(\begin{array}{lll}0 & 0 & 1 \\ 0 & 1 & 0 \\ 1 & 0 & 0\end{array}\right)$ from the left and by $\left(\begin{array}{rrr}0 & 1 & 0 \\ 0 & 0 & -1 \\ 1 & 0 & 0\end{array}\right)$ from the right and after passing from $z_{1}$ and $z_{3}$ to $\bar{z}_{1}$ and $\bar{z}_{3}$ we obtain the map $\eta$ of the next section. There, we introduce the resulting map directly by the complex cross product. For $n \geq 4$ we do not 
know whether any of the maps $\eta_{n}$ or any map homotopic to some $\eta_{n}$ has any nice geometric properties or can be found by a more geometric construction. Note that an elementary induction shows that all $\eta_{n}$ have the property that $\overline{\eta_{n}(z)}=\eta_{n}(\bar{z})$ for all $z \in \mathbb{S}^{2 n-1}$.

We now point out a property of the generators of $\pi_{2 n-1} \mathrm{U}(n)$ that will be the key for understanding the first nonstable groups $\pi_{2 n} \mathrm{U}(n) \approx \mathbb{Z}_{n}$ ! in Section 3 . Given a $\operatorname{map} \theta: \mathbb{S}^{2 n-1} \rightarrow \mathrm{U}(n)$ we obtain a map $p_{j} \circ \theta: \mathbb{S}^{2 n-1} \rightarrow \mathbb{S}^{2 n-1}$ using the projection $p_{j}: \mathrm{U}(n) \rightarrow \mathbb{S}^{2 n-1}$ that maps a matrix to its $j$-th column.

Lemma 1.1. The assignment

$$
\theta \mapsto \frac{1}{(n-1) !} \operatorname{deg}\left(p_{j} \circ \theta\right)
$$

yields an isomorphism $\pi_{2 n-1} \mathrm{U}(n) \rightarrow \mathbb{Z}$. This isomorphism is independent of $j$.

In other words, a map $\theta: \mathbb{S}^{2 n-1} \rightarrow \mathrm{U}(n)$ represents a generator of $\pi_{2 n-1} \mathrm{U}(n)$ if and only if the composition with the projection to some (and hence any) of the columns has degree $\pm(n-1)$ ! where the sign is independent of the column.

Proof. The first part follows immediately from the exact homotopy sequence of the bundle $\mathrm{U}(n-1) \rightarrow \mathrm{U}(n) \rightarrow \mathbb{S}^{2 n-1}$ using the fact that $\pi_{2 n-2} \mathrm{U}(n-1) \approx \mathbb{Z}_{(n-1)}$ ! and stable homotopy groups. In order to see that, say, $p_{n-1}$ and $p_{n}$ yield the same isomorphism, we multiply the values of $\theta$ from the right by the matrix in (2) with $t=\frac{\pi}{2}$. The resulting map $\theta^{\prime}$ is homotopic to $\theta$ and we get $p_{n-1} \circ \theta^{\prime}=p_{n} \circ \theta$.

\section{A minimal generator of $\pi_{5} \mathrm{SU}(3)$}

Given two vectors $z, w \in \mathbb{C}^{3}$ their cross product is defined to be

$$
z \times w=\left(\begin{array}{c}
\bar{z}_{2} \bar{w}_{3}-\bar{z}_{3} \bar{w}_{2} \\
\bar{z}_{3} \bar{w}_{1}-\bar{z}_{1} \bar{w}_{3} \\
\bar{z}_{1} \bar{w}_{2}-\bar{z}_{2} \bar{w}_{1}
\end{array}\right)
$$

If $z$ and $w$ are unit vectors that are perpendicular with respect to the standard hermitian inner product on $\mathbb{C}^{3}$ then $z \times w$ is the unique vector such that the matrix whose columns are $z, w$, and $z \times w$ is contained in $\mathrm{SU}(3)$. Hence,

$$
(A \cdot z) \times(A \cdot w)=A \cdot(z \times w)
$$

for all $A \in \mathrm{SU}(3)$ and $z, w \in \mathbb{C}^{3}$.

We can now define an embedding $\eta: \mathbb{S}^{5} \rightarrow \mathrm{SU}(3)$ by setting $\eta(z) \cdot \bar{z}=z$ and $\eta(z) \cdot \bar{w}=z \times w$ if $w$ is perpendicular to $z$. This map is obviously not nullhomotopic. For if it were homotopic to the constant map from $\mathbb{S}^{5}$ to the identity in $\mathrm{SU}(3)$ then the map from $\mathbb{S}^{5}$ to itself given by complex conjugation would be homotopic to the identity map of $\mathbb{S}^{5}$, which is not true. An explicit formula for $\eta$ 
is given as follows:

$$
\eta(z)=z z^{t}+\left(\begin{array}{rrr}
0 & -\bar{z}_{3} & \bar{z}_{2} \\
\bar{z}_{3} & 0 & -\bar{z}_{1} \\
-\bar{z}_{2} & \bar{z}_{1} & 0
\end{array}\right)
$$

Theorem 2.1. The embedding $\eta: \mathbb{S}^{5} \rightarrow \mathrm{SU}(3)$ generates $\pi_{5} \mathrm{SU}(3) \approx \mathbb{Z}$.

Proof. If we compose $\eta$ with the projection to any of the columns of SU(3) we obtain a map from $\mathbb{S}^{5}$ to itself with degree 2. It follows from Lemma 1.1 that $\eta$ represents a generator of $\pi_{5} \mathrm{SU}(3)$.

It follows from property (3) that $\eta$ is equivariant with respect to the standard action of $\mathrm{SU}(3)$ on $\mathbb{S}^{5} \subset \mathbb{C}^{3}$ and the action of $\mathrm{SU}(3)$ on itself given as follows:

$$
\mathrm{SU}(3) \times \mathrm{SU}(3) \rightarrow \mathrm{SU}(3), \quad(B, A) \mapsto B A B^{t} .
$$

It is known that the orbit space of the latter action is a closed interval. In order to give a more detailed description of the orbit structure we use the geodesic

$$
c(t)=\left(\begin{array}{llr}
1 & 0 & 0 \\
0 & \cos t & -\sin t \\
0 & \sin t & \cos t
\end{array}\right)
$$

The orbit through $c(0)=\mathbb{1}$ is diffeomorphic to the symmetric space $\mathrm{SU}(3) / \mathrm{SO}(3)$ and consists precisely of the symmetric matrices in $\mathrm{SU}(3)$. It is easy to see that $c$ intersects this orbit perpendicularly (and hence all orbits by Clairault's theorem that the velocity vectors of a geodesic have a constant inner product with a Killing field). The orbits through $c(t)$ for $t \in] 0, \frac{\pi}{2}[$ are diffeomorphic to the seven-dimensional space $\mathrm{SU}(3) / \mathrm{SO}(2)$. Finally, we have

$$
c\left(\frac{\pi}{2}\right)=\left(\begin{array}{rrr}
1 & 0 & 0 \\
0 & 0 & -1 \\
0 & 1 & 0
\end{array}\right)=\eta\left(\begin{array}{l}
1 \\
0 \\
0
\end{array}\right) .
$$

Hence, $\eta$ parametrizes the isolated singular orbit through $c\left(\frac{\pi}{2}\right)$. Since isolated orbits are minimal submanifolds (in the usual sense that they are critical points for the volume functional, i.e., their mean curvature vanishes) [11], we get:

Proposition 2.2. The embedding $\eta$ parametrizes a minimal submanifold of $\mathrm{SU}(3)$.

We finally mention the following curiosity: Using the embedding $\eta$, the Hopf fibration $\mathbb{S}^{5} \rightarrow \mathbb{C P}^{2}$ can be extended to a simple self-map of SU(3), namely, to the map $A \mapsto A \cdot \bar{A}$. Indeed, if we multiply $\eta$ and $\bar{\eta}$ value by value we obtain the map

$$
\mathbb{S}^{5} \rightarrow \mathrm{SU}(3), \quad z \mapsto 2 z \bar{z}^{t}-\mathbb{1} .
$$

This map is the standard totally geodesic Cartan embedding of $\mathbb{C P}^{2}$ into $\mathrm{SU}(3)$. It follows from Lemma 5.1 or by inspecting the orbit space of the adjoint action of $\mathrm{SU}(3)$ that $\eta \cdot \bar{\eta}$ is null-homotopic. 


\section{The first nonstable homotopy groups $\pi_{2 n} \mathrm{SU}(n) \approx \mathbb{Z}_{n}$ !}

Bott [2] showed in 1958 that the image of $\pi_{2 n} \mathrm{BU}$ in $H_{2 n}(\mathrm{BU})$ is divisible by precisely $(n-1)$ !. This refined the previous result of Borel and Hirzebruch [1] that these classes are divisible by $(n-1)$ ! except for the prime 2 . As a consequence of the refined version, the first nonstable homotopy groups $\pi_{2 n} \mathrm{U}(n)$ of the unitary groups are isomorphic to the cyclic groups of order $n$ !. This result was used almost immediately by Kervaire [13] and Milnor [4] who independently gave the first proofs of the fact that the only parallelizable spheres are $\mathbb{S}^{1}, \mathbb{S}^{3}$, and $\mathbb{S}^{7}$. Generators of the groups $\pi_{2 n} \mathrm{U}(n)$ are represented by the characteristic maps of the bundles $\mathrm{U}(n+1) \rightarrow \mathbb{S}^{2 n+1}$. These maps were known explicitly several years before Bott's result [21]. We will deform them in a way that allows us to see how they become null-homotopic in the $n$ !-th power.

The group $\mathrm{SU}(n)$ acts transitively on the unit sphere $\mathbb{S}^{2 n-1}$ in $\mathbb{C}^{n}$. The isotropy group of the $j$-th canonical basis vector in $\mathbb{C}^{n}$ is denoted by $\mathrm{SU}(n-1)_{j}$. It is the subgroup of $\mathrm{SU}(n)$ whose $j$-th diagonal entry is 1 . Natural diffeomorphisms between $\mathrm{SU}(n) / \mathrm{SU}(n-1)_{j}$ and $\mathbb{S}^{2 n-1}$ are given by the projections $p_{j}: \mathrm{SU}(n) \rightarrow$ $\mathbb{S}^{2 n-1}$ that map matrices to their $j$-th columns. Now consider the maps

$$
\phi_{j}:\left[0, \frac{2 \pi}{n}\right] \times \mathrm{SU}(n) / \mathrm{SU}(n-1)_{j} \longrightarrow \mathrm{SU}(n)
$$

given by

$$
\begin{gathered}
\phi_{1}(t, A)=A \cdot \operatorname{diag}\left(e^{i(n-1) t}, e^{-i t}, \ldots, e^{-i t}\right) \cdot A^{-1} \\
\vdots \\
\phi_{n}(t, A)=A \cdot \operatorname{diag}\left(e^{-i t}, \ldots, e^{-i t}, e^{i(n-1) t}\right) \cdot A^{-1} .
\end{gathered}
$$

For $t=0$ and $t=\frac{2 \pi}{n}$ the values of all $\phi_{j}$ are independent of $A \in \mathrm{SU}(n)$. Hence the $\phi_{j}$ induce maps $\mathbb{S}^{2 n} \rightarrow \mathrm{SU}(n)$.

Lemma 3.1. All the maps $\phi_{j}$ above induce the same map $\phi: \mathbb{S}^{2 n} \rightarrow \mathrm{SU}(n)$. This map represents a generator of $\pi_{2 n} \mathrm{SU}(n)$.

Proof. Consider a matrix $A$ whose first column is given by $z \in \mathbb{S}^{2 n-1}$. Then

$$
\begin{aligned}
A \cdot \operatorname{diag}\left(e^{i(n-1) t}, e^{-i t}, \ldots, e^{-i t}\right) \cdot A^{-1} & \\
=A \cdot e^{-i t} \cdot\left(\mathbb{1}+\operatorname{diag}\left(e^{i n t}-1,0, \ldots, 0\right)\right) \cdot A^{-1} & =e^{-i t}\left(\mathbb{1}+z\left(e^{i n t}-1\right) \bar{z}^{t}\right) .
\end{aligned}
$$

For the other columns the computation is analogous and yields the same result. We compose the map

$$
\hat{\phi}:\left[0, \frac{2 \pi}{n}\right] \times \mathbb{S}^{2 n-1} \rightarrow \mathrm{SU}(n), \quad(t, z) \mapsto e^{-i t}\left(\mathbb{1}+z\left(e^{i n t}-1\right) \bar{z}^{t}\right)
$$


with the inverse of the suspension

$$
\left[0, \frac{2 \pi}{n}\right] \times \mathbb{S}^{2 n-1} \rightarrow \mathbb{S}^{2 n} \subset \mathbb{R} \times \mathbb{C}^{n}, \quad(t, z) \mapsto\left(\frac{t n}{\pi}-1, z \sqrt{1-\left(\frac{t n}{\pi}-1\right)^{2}}\right) .
$$

This yields the map

$$
\phi: \mathbb{S}^{2 n} \rightarrow \mathrm{SU}(n), \quad(y, z) \mapsto e^{-i \pi(y+1) / n} \cdot\left(\mathbb{1}-\frac{z}{|z|}\left(1+e^{i \pi y}\right) \frac{\bar{z}^{t}}{|z|}\right) .
$$

We can remove the factor in front of the paranthesis and obtain a homotopic map with values in $\mathrm{U}(n)$. Moreover, we can substitute the rational parametrization $\left(\frac{1+i y}{1-i y}\right)^{2}$ of the unit circle in $\mathbb{C}$ for the exponential parametrization $e^{i \pi y}$ without changing the homotopy class of $\phi$. This leads to the map

$$
\mathbb{S}^{2 n} \rightarrow \mathrm{U}(n), \quad(y, z) \mapsto \mathbb{1}-2 z \frac{1}{(1-i y)^{2}} \bar{z}^{t} .
$$

In Steenrod's book [21] it is proved that this map represents the characteristic map of the bundle $\mathrm{U}(n+1) \rightarrow \mathbb{S}^{2 n+1}$ and hence a generator of $\pi_{2 n} \mathrm{U}(n)$.

At first glance it might seem like one could multiply the $n$ maps $\phi_{1}, \ldots, \phi_{n}$ value by value and the result is the constant map to the identity. This would imply that $\pi_{2 n} \mathrm{SU}(n)$ is of order at most $n$ contradicting $\pi_{2 n} \mathrm{SU}(n) \approx \mathbb{Z}_{n !}$. The reason why this does not work is that we are not multiplying maps that have the same domain of definition, since the isotropy groups $\mathrm{SU}(n-1)_{j}$ are different. In order to get maps from the same domain of definition $\left[0, \frac{2 \pi}{n}\right] \times \mathbb{S}^{2 n-1}$ one has to use the identifications between $\mathrm{SU}(n) / \mathrm{SU}(n-1)_{j}$ and $\mathbb{S}^{2 n-1}$. But, as we saw, this always yields the same map $\phi$ above and $\phi^{n}$ is evidently not the constant map to the identity.

There is, however, a way to make the previous idea work. The clue is to use any map $\eta: \mathbb{S}^{2 n-1} \rightarrow \mathrm{SU}(n)$ that represents a generator of the stable group $\pi_{2 n-1} \mathrm{SU}(n)$. Such a map has the fundamental property that the composition $p_{j} \circ \eta$ with the projection $p_{j}$ to the $j$-th matrix column has degree $\pm(n-1)$ ! where the sign is independent of the column (see Lemma 1.1). We now obtain maps

$$
\psi_{j}:\left[0, \frac{2 \pi}{n}\right] \times \mathbb{S}^{2 n-1} \rightarrow \mathrm{SU}(n)
$$

by plugging $p_{j} \circ \eta$ into the second argument of $\hat{\phi}$, i.e., by

$$
\begin{gathered}
\psi_{1}(t, z)=\phi_{1}(t, \eta(z))=\eta(z) \cdot \operatorname{diag}\left(e^{i(n-1) t}, e^{-i t}, \ldots, e^{-i t}\right) \cdot \eta(z)^{-1} \\
\vdots \\
\psi_{n}(t, z)=\phi_{n}(t, \eta(z))=\eta(z) \cdot \operatorname{diag}\left(e^{-i t}, \ldots, e^{-i t}, e^{i(n-1) t}\right) \cdot \eta(z)^{-1} .
\end{gathered}
$$

The following is now evident and shows us explicitly how the $n$ !-th power of a generator of $\pi_{2 n} \mathrm{SU}(n)$ is null-homotopic.

Theorem 3.2. The maps $\psi_{j}$ induce maps $\mathbb{S}^{2 n} \rightarrow \mathrm{SU}(n)$ that represent $(n-1)$ ! times the same generator of $\pi_{2 n} \mathrm{SU}(n)$. The maps $\psi_{j}$ commute mutually and their product $\psi_{1} \cdot \ldots \cdot \psi_{n}$ is the constant map to the identity. 
Explicit homotopies between the maps $\psi_{j}$ are easily given. The formula

$$
\eta(z) \cdot\left(\begin{array}{ccc}
\cos s & -\sin s & 0 \\
\sin s & \cos s & 0 \\
0 & 0 & 11
\end{array}\right) \cdot \operatorname{diag}\left(e^{i(n-1) t}, e^{-i t}, \ldots, e^{-i t}\right) \cdot\left(\begin{array}{ccc}
\cos s & \sin s & 0 \\
-\sin s & \cos s & 0 \\
0 & 0 & 11
\end{array}\right) \cdot \eta(z)^{-1},
$$

for example, yields the map $\psi_{1}$ for $s=0$ and the map $\psi_{2}$ for $s=\frac{\pi}{2}$.

Remark 3.3. Theorem 3.2 and Lemma 1.1 together provide inductively an elementary proof for the fact that $\pi_{2 n} \mathrm{SU}(n)$ is a cyclic group whose order divides $n$ !. In order to show that $n$ ! divides the order of $\pi_{2 n} \mathrm{SU}(n)$, however, cohomological arguments like those in [2] seem to be inevitable.

Remark 3.4. The map $\hat{\phi}$ factors through a map $\check{\phi}$ defined on $\left[0, \frac{2 \pi}{n}\right] \times \mathbb{C P}^{n-1}$. The $\mathbb{C} \mathbb{P}^{n-1}$ can be considered to represent the space of shortest curves from the identity matrix $\mathbb{1}$ to the matrix $e^{-2 \pi i / n} \cdot \mathbb{1}$ in the center of $\mathrm{SU}(n)$. The map $\check{\phi}$ appears in Bott's papers [2], [3] frequently, but not with the meaning that it provides a generator of the group $\pi_{2 n} \mathrm{SU}(n)$.

\section{The homotopy groups $\pi_{2 n} \mathrm{SU}(n-1)$}

The homotopy groups $\pi_{2 n} \mathrm{SU}(n-1)$ were first computed by Kervaire [14]. The following fact is central for the computation: Given a generator $\phi$ of $\pi_{2 n} \mathrm{SU}(n)$ the composition $p_{j} \circ \phi: \mathbb{S}^{2 n} \rightarrow \mathbb{S}^{2 n-1}$ with the projection to the $j$-th column is null-homotopic if $n$ is odd and homotopic to the $(2 n-3)$-rd suspension of the Hopf fibration $\mathbb{S}^{3} \rightarrow \mathbb{S}^{2}$ if $n$ is even (see [14], [21]). With this fact Kervaire [14] deduced from the exact homotopy sequence of the bundle $\mathrm{SU}(n) \rightarrow \mathbb{S}^{2 n-1}$ that $\pi_{2 n-1} \mathrm{SU}(n-1)$ is trivial if $n$ is even and isomorphic to $\mathbb{Z}_{2}$ if $n$ is odd and that

$$
\pi_{2 n} \mathrm{SU}(n-1) \approx \begin{cases}\mathbb{Z}_{n ! / 2} & \text { if } n \text { is even, } \\ \mathbb{Z}_{2} \oplus \mathbb{Z}_{n !} & \text { if } n \geq 5 \text { is odd } \\ \mathbb{Z}_{12} & \text { if } n=3 .\end{cases}
$$

In other words, if $n$ is odd, $\phi$ is homotopic to a map with values in $\mathrm{SU}(n-1)$ (it is, however, not very easy to write this homotopy down explicitly). If $n=2 m$ is even, $\phi$ cannot be deformed to a map with values in $\mathrm{SU}(n-1)$, but $\phi^{2}$ can, and the resulting map represents a generator of $\pi_{4 m} \mathrm{SU}(2 m-1)$. We will now describe this deformation explicitly by reducing the equivariance group of $\phi$ from $\mathrm{SU}(2 m)$ to $\operatorname{Sp}(m)$.

The symplectic group $\mathrm{Sp}(m)$ can be regarded as the subgroup of matrices $A \in \mathrm{SU}(2 m)$ with $A^{t} J A=J$. Here, $J \in \mathrm{SU}(2 m)$ is the matrix whose diagonal $2 \times 2$-blocks are $\left(\begin{array}{rr}0 & -1 \\ 1 & 0\end{array}\right)$. A matrix $A \in \mathrm{SU}(2 m)$ with columns $v_{1}, \ldots, v_{2 m}$ belongs to $\operatorname{Sp}(m)$ if and only if $v_{2 k}=J \cdot \bar{v}_{2 k-1}$ for all $k=1, \ldots, m$. The group $\operatorname{Sp}(m)$ acts transitively on the unit sphere $\mathbb{S}^{4 m-1}$ in $\mathbb{C}^{2 m}$. The isotropy groups of the first and the second canonical basis vector in $\mathbb{C}^{2 m}$ are the same, namely, the subgroup 
$\operatorname{Sp}(m-1)_{1}$ of matrices in $\operatorname{Sp}(m)$ whose first and second diagonal entry is 1 . Now we consider the maps $\phi_{1}$ and $\phi_{2}$ of the previous section and restrict the second argument of these maps to symplectic matrices. This way we obtain maps

$$
\phi_{1}^{\prime}, \phi_{2}^{\prime}:\left[0, \frac{\pi}{m}\right] \times \mathrm{Sp}(m) / \mathrm{Sp}(m-1)_{1} \rightarrow \mathrm{SU}(2 m)
$$

with the same domain of definition. Both these maps still induce the generator $\phi: \mathbb{S}^{4 m} \rightarrow \mathrm{SU}(2 m)$ of $\pi_{4 m} \mathrm{SU}(2 m)$ given in the previous section. Their product is the map

$$
\begin{aligned}
\phi_{12}^{\prime}:\left[0, \frac{\pi}{m}\right] \times \operatorname{Sp}(m) / \operatorname{Sp}(m-1)_{1} & \rightarrow \mathrm{SU}(2 m), \\
(t, A) & \mapsto A \cdot \operatorname{diag}\left(e^{i(2 m-2) t}, e^{i(2 m-2) t}, e^{-2 i t}, \ldots, e^{-2 i t}\right) \cdot A^{-1} .
\end{aligned}
$$

Lemma 4.1. The map $\phi_{12}^{\prime}$ and the analogously defined maps $\phi_{34}^{\prime}, \ldots, \phi_{2 m-1,2 m}^{\prime}$ all induce the same map $\phi^{(2)}: \mathbb{S}^{4 m} \rightarrow \mathrm{SU}(2 m)$ which represents twice a generator of $\pi_{4 m} \mathrm{SU}(2 m)$. The deformation to $\mathrm{SU}(2 m-1)$ of Section 1.3 can be applied to $\phi^{(2)}$ and the deformed map represents a generator of $\pi_{4 m} \mathrm{SU}(2 m-1)$.

Proof. Since $\phi_{12}^{\prime}$ is the product of $\phi_{1}^{\prime}$ and $\phi_{2}^{\prime}$ it is evident that $\phi^{(2)}$ represents twice a generator of $\pi_{4 m} \mathrm{SU}(2 m)$. Computations analogous to that of the previous section show the following: If the first column of the matrix $A$ is the vector $z \in \mathbb{C}^{2 m}$ then

$$
\phi_{12}^{\prime}(t, A)=e^{-2 i t}\left(\mathbb{1}+\left(e^{2 m i t}-1\right)\left(z \bar{z}^{t}-J \bar{z} z^{t} J\right)\right)
$$

and the map $\phi_{12}^{\prime}$ induces the map

$$
\begin{aligned}
& \phi^{(2)}: \mathbb{S}^{4 m} \rightarrow \mathrm{SU}(2 m), \\
&(y, z) \mapsto e^{-i \pi(y+1) / m} \cdot\left(\mathbb{1}-\left(1+e^{i \pi y}\right)\left(\frac{z}{|z|} \frac{\bar{z}^{t}}{|z|}-J \frac{\bar{z}}{|z|} \frac{z^{t}}{|z|} J\right)\right) .
\end{aligned}
$$

The $(2,1)$-entry in the values of $\phi^{(2)}$ is always zero. Hence, after multiplying $\phi^{(2)}$ from the left and the right with suitable permutation matrices, the lower right entry vanishes and the deformation of Section 1.3 can be applied.

Analogously to the previous section we can plug a generator of $\pi_{4 m-1} \operatorname{Sp}(m) \approx$ $\mathbb{Z}$ into the second argument of the maps $\phi_{12}^{\prime}, \ldots, \phi_{2 m-1,2 m}^{\prime}$. The resulting maps will be denoted by $\psi_{12}^{\prime}, \ldots, \psi_{2 m-1,2 m}^{\prime}$. Like $\phi_{12}^{\prime}$ they can all be deformed to $\mathrm{SU}(2 m-1)$ with the explicit deformation of Section 1.3.

Proposition 4.2. The $m$ maps $\psi_{2 k-1,2 k}^{\prime}$ induce maps $\mathbb{S}^{4 m} \rightarrow \mathrm{SU}(2 m-1)$ that represent $(2 m-1)$ ! times a generator of $\pi_{4 m} \mathrm{SU}(2 m-1)$ if $m$ is odd and $2 \cdot(2 m-1)$ ! times a generator if $m$ is even. They commute and their product is the constant map to the identity.

Proof. It follows from the exact homotopy sequence of the bundle $\operatorname{Sp}(m) \rightarrow \mathbb{S}^{4 m-1}$ that the composition of a generator of $\pi_{4 m-1} \mathrm{Sp}(m-1)$ with the projection to any of the columns of $\operatorname{Sp}(m-1)$ yields a self-map of $\mathbb{S}^{4 m-1}$ whose degree is the order 
of the cyclic group $\pi_{4 m-2} \operatorname{Sp}(m-1)$. Kervaire [15] first showed that the order of this group is $(2 m-1)$ ! if $m$ is odd and $2 \cdot(2 m-1)$ ! if $m$ is even.

Remark 4.3. The map $\phi^{(2)}$ is homotopic to the map

$$
\mathbb{S}^{4 m} \rightarrow \mathrm{U}(2 m), \quad(y, z) \mapsto \mathbb{1}-\frac{2}{(1-i y)^{2}}\left(z \bar{z}^{t}-J \bar{z} z^{t} J\right)
$$

with values in $\mathrm{U}(2 m)$.

Remark 4.4. The map $\phi_{12}^{\prime}$ factors through a map defined on

$$
\left[0, \frac{\pi}{m}\right] \times \operatorname{Sp}(m) /(\operatorname{Sp}(m-1) \times \operatorname{Sp}(1)) .
$$

In the case $m=1$ the factor on the right is trivial and the maps $\phi_{12}^{\prime}$ and $\phi^{(2)}$ are the constant maps to the identity. In the case $m=2$ the factor on the right is diffeomorphic to $\mathbb{S}^{4}$ and hence $\phi_{12}^{\prime}$ induces a map $\mathbb{S}^{5} \rightarrow \mathrm{SU}(4)$. It is not difficult to see that this is the map $\zeta_{3}$ from Section 1 . This means that a generator of $\pi_{8} \mathrm{SU}(3)$ is given by composing the first suspension of the Hopf fibration $\mathbb{S}^{7} \rightarrow \mathbb{S}^{4}$ with the generator of $\pi_{5} \mathrm{SU}(3)$ described in Section 2 .

\section{Symmetric maps into the unitary groups and homotopy groups of $\mathrm{Sp}(n)$ and $\mathrm{SU}(n) / \mathrm{SO}(n)$}

The Cartan embedding of the symmetric space $\mathrm{SU}(n) / \mathrm{SO}(n)$ into the Lie group $\mathrm{SU}(n)$ is the map

$$
\mathcal{C}: \mathrm{SU}(n) / \mathrm{SO}(n) \rightarrow \mathrm{SU}(n), \quad A \cdot \mathrm{SO}(n) \mapsto A \cdot A^{t} .
$$

The image of this map is precisely the space of symmetric matrices in $\mathrm{SU}(n)$. We combine this fact with the explicit form of the Bott periodicity isomorphism given in Section 1. This combination provides first maps that represent non-trivial elements of certain homotopy groups of the symplectic groups and second a structure theorem for certain nonstable homotopy groups of $\mathrm{SU}(n) / \mathrm{SO}(n)$.

We begin with the following statement:

Lemma 5.1. Any map $\mathbb{S}^{2 k-1} \rightarrow \mathrm{U}(n)$ with $k \leq n$ is homotopic to its transposed if $k$ is odd and homotopic to its complex conjugate if $k$ is even. Any map from $\mathbb{S}^{2 n}$ to $\mathrm{U}(n)$ or $\mathrm{U}(n-1)$ is homotopic to its transposed if $n$ is even and homotopic to its complex conjugate if $n$ is odd.

Proof. Complex conjugation on $\mathrm{SU}(k)$ corresponds to complex conjugation on the sphere $\mathbb{S}^{2 k-1} \subset \mathbb{C}^{k}$ under the projection $p_{1}: \mathrm{SU}(k) \rightarrow \mathbb{S}^{2 k-1}$. The complex conjugation on $\mathbb{S}^{2 k-1}$ is homotopic to the identity map if $k$ is even and not homotopic to the identity map if $k$ is odd. The lemma follows now from the following part of 
the exact homotopy sequence

$$
\begin{aligned}
& \pi_{2 k-1} \mathrm{SU}(k) \longrightarrow \pi_{2 k-1} \mathbb{S}^{2 k-1} \longrightarrow \pi_{2 k-2} \mathrm{SU}(k-1) \longrightarrow \pi_{2 k-2} \mathrm{SU}(k)
\end{aligned}
$$

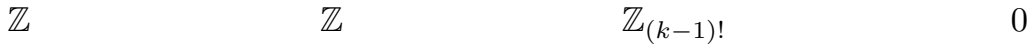

and from the fact that a generator of the homotopy group $\pi_{2 n} \mathrm{U}(n-1)$ is homotopic to a generator of $\pi_{2 n} \mathrm{U}(n)$ or to twice a generator or of order 2 (see Section 4 ).

Note that in $\mathrm{SU}(2)$ complex conjugation is an inner automorphism and thus any non-trivial map from any sphere $\mathbb{S}^{m}$ to $\mathrm{SU}(2)$ is homotopic to its conjugate and therefore only homotopic to its transposed if it is of order 2 . From the higher homotopy groups of $\mathrm{SU}(2) \approx \mathbb{S}^{3}$ it is now clear that there exist many maps $\mathbb{S}^{2 k-1} \rightarrow$ $\mathrm{SU}(2)$ with odd $k$ that are not homotopic to their transposed.

Let $\mathrm{Sp}(n) \subset \mathrm{SU}(2 n)$ be the subgroup of matrices of the form $\left(\begin{array}{c}A-\bar{B} \\ B\end{array} \bar{A}\right)$ (note that this subgroup of $\mathrm{SU}(2 n)$ agrees with the $\operatorname{Sp}(n)$ used in Section 4 only up to conjugation). The following property of the explicit form of the Bott periodicity isomorphism $\mathcal{B}$ given in (1.1) is now apparent.

Lemma 5.2. Let $\theta$ be a symmetric map $\mathbb{S}^{2 k-1} \rightarrow \mathrm{U}(n)$, i.e., $\theta(z)^{t}=\theta(z)$ for all $z \in \mathbb{S}^{2 k-1}$. Then $\mathcal{B}(\theta)$ takes values in $\operatorname{Sp}(n) \subset \mathrm{SU}(2 n)$.

Corollary 5.3. Any symmetric map $\theta: \mathbb{S}^{2 k-1} \rightarrow \mathrm{U}(n)$ with even $k \leq n$ is nullhomotopic.

Proof. If $\theta$ would represent a non-trivial element in the stable group $\pi_{2 k-1} \mathrm{U}(n)$, then $\mathcal{B}(\theta)$ would represent a non-trivial element in $\pi_{2 k+1} \mathrm{SU}(2 n) \approx \mathbb{Z}$. But $\mathcal{B}(\theta)$ takes values in $\operatorname{Sp}(n)$ and the stable group $\pi_{2 k+1} \operatorname{Sp}(n)$ is trivial or isomorphic to $\mathbb{Z}_{2}$ if $k$ is even.

Proposition 5.4. If $\theta$ is a generator of $\pi_{2 k-1} \mathrm{U}(n)$ with $k=2 m+1 \leq n$ then $\mathcal{B}\left(\theta \cdot \theta^{t}\right)$ represents a generator of $\pi_{2 k+1} \mathrm{Sp}(n) \approx \mathbb{Z}$ if $m$ is odd and twice a generator if $m$ is even.

Proof. Because of Lemma 5.1, $\theta \cdot \theta^{t}$ represents in $\pi_{2 k-1} \mathrm{U}(n)$ twice the generator given by $\theta$. Correspondingly, $\mathcal{B}\left(\theta \cdot \theta^{t}\right)$ represents twice a generator of $\pi_{2 k+1} \mathrm{SU}(2 n)$. Since $\theta \cdot \theta^{t}$ is symmetric, $\mathcal{B}\left(\theta \cdot \theta^{t}\right)$ falls into $\operatorname{Sp}(n)$. We now inspect part of the exact homotopy sequence of the bundle that belongs to the homogeneous space $\mathrm{SU}(2 n) / \operatorname{Sp}(n)$ :

$$
\pi_{2 k+1} \mathrm{Sp}(n) \rightarrow \pi_{2 k+1} \mathrm{SU}(2 n) \rightarrow \pi_{2 k+1}(\mathrm{SU}(2 n) / \mathrm{Sp}(n)) \rightarrow \pi_{2 k} \mathrm{Sp}(n) .
$$

All the homotopy groups involved here are stable, the first two isomorphic to $\mathbb{Z}$, the last one trivial, and $\pi_{2 k+1}(\mathrm{SU}(2 n) / \mathrm{Sp}(n))$ trivial if $m$ is even and isomorphic to $\mathbb{Z}_{2}$ if $m$ is odd. 
Corollary 5.5. A generator of the stable group $\pi_{2 k-1} \mathrm{U}(n)$ with $k=4 l+3 \leq n$ cannot be represented by a symmetric map.

We will now apply the statements above to determine the structure of the semistable homotopy groups $\pi_{8 l+5}(\mathrm{SU}(n) / \mathrm{SO}(n))$ for $l \leq \frac{n-3}{4}$. Given a map $\theta: \mathbb{S}^{2 k-1} \rightarrow \mathrm{SU}(n)$ with odd $k$, the composition

$$
\mathbb{S}^{2 k-1} \stackrel{\theta}{\longrightarrow} \mathrm{SU}(n) \rightarrow \mathrm{SU}(n) / \mathrm{SO}(n) \stackrel{\mathcal{C}}{\longrightarrow} \mathrm{SU}(n) \cap \operatorname{Sym}(n, \mathbb{C}) \hookrightarrow \mathrm{SU}(n)
$$

yields the symmetric map $\theta \cdot \theta^{t}$ which represents twice the element in $\pi_{2 k-1} \mathrm{SU}(n)$ that is represented by $\theta$. Hence, if $\theta$ represents a generator of $\pi_{2 k-1} \mathrm{SU}(n)$ then $\theta \cdot \theta^{t}$ represents a generator or twice a generator of a $\mathbb{Z}$-factor in

$$
\pi_{2 k-1}(\mathrm{SU}(n) \cap \operatorname{Sym}(n, \mathbb{C})) \approx \pi_{2 k-1}(\mathrm{SU}(n) / \mathrm{SO}(n)) .
$$

This ambiguity remains in the case $k=4 l+1 \leq n$ as we shall see below. However, if $k=4 l+3 \leq n$ then $\theta \cdot \theta^{t}$ cannot represent twice a generator because of the previous corollary.

Theorem 5.6. If $3 \leq k=4 l+3 \leq n$ then a generator of $\pi_{2 k-1} \mathrm{SU}(n)$ projects to a generator of a $\mathbb{Z}$-factor in $\pi_{2 k-1}(\mathrm{SU}(n) / \mathrm{SO}(n))$. Consequently we have

$$
\begin{aligned}
& \pi_{8 l+5}(\mathrm{SU}(n) / \mathrm{SO}(n)) \approx \mathbb{Z} \oplus \pi_{8 l+4} \mathrm{SO}(n) \text { and } \\
& \pi_{8 l+6}(\mathrm{SU}(n) / \mathrm{SO}(n)) \approx \pi_{8 l+5} \mathrm{SO}(n) .
\end{aligned}
$$

Proof. The first part follows from the factorization (4) of the map $\theta \cdot \theta^{t}$, the second part from the first and the relevant segment of the exact homotopy sequence of the bundle $\mathrm{SO}(n) \rightarrow \mathrm{SU}(n) \rightarrow \mathrm{SU}(n) / \mathrm{SO}(n)$.

This statement was obtained before by Kachi (see [12], Proposition 3.5) for $7 \leq k=4 l+3 \leq n-1$. Kachi's proof is based on computations of Kervaire [14]. These, in turn, involve certain homotopy groups of the Stiefel manifolds that were determined by Paechter [20]. Our proof, on the other hand, requires just the knowledge of stable homotopy groups.

The simplest example where our statement provides information is the homotopy group $\pi_{5}(\mathrm{SU}(3) / \mathrm{SO}(3))$. The exact homotopy sequence leaves the two choices $\mathbb{Z}$ and $\mathbb{Z} \oplus \mathbb{Z}_{2}$. Our argument above shows that $\pi_{5}(\mathrm{SU}(3) / \mathrm{SO}(3))$ is isomorphic to the second group.

Proposition 5.7. If $5 \leq k=4 l+1 \leq n$ then a generator of $\pi_{2 k-1} \mathrm{SU}(n)$ can only project to a generator or to twice a generator of a $\mathbb{Z}$-factor in $\pi_{2 k-1}(\mathrm{SU}(n) / \mathrm{SO}(n))$. In the first case we have

$$
\pi_{8 l+1}(\mathrm{SU}(n) / \mathrm{SO}(n)) \approx \mathbb{Z} \oplus \pi_{8 l} \mathrm{SO}(n) .
$$

In the second case, $\pi_{8 l} \mathrm{SO}(n)$ is isomorphic to a direct sum $G \oplus \mathbb{Z}_{2}$ such that

$$
\pi_{8 l+1}(\mathrm{SU}(n) / \mathrm{SO}(n)) \approx \mathbb{Z} \oplus G .
$$


In any of the two cases we have

$$
\pi_{8 l+2}(\mathrm{SU}(n) / \mathrm{SO}(n)) \approx \pi_{8 l+1} \mathrm{SO}(n)
$$

In the stable range obviously the second alternative holds. On the other hand, the group $\pi_{8} \mathrm{SO}(5)$ (which occurs in the first case covered by the proposition) is trivial. For information on $\pi_{n+r}(\mathrm{SU}(n) / \mathrm{SO}(n))$ for $n \geq 8$ and $r \leq 5$ we refer to $[12]$.

\section{Appendix. The first homotopy groups of the unitary groups}

For the convenience of the reader we provide in Table 1 the very first homotopy groups of the unitary groups. Larger tables can be found in [17]. The black line in the table indicates the border between the stable and the nonstable groups. A $t$ or $c$ below a group $\pi_{r} \mathrm{U}(n)$ indicates that any map $\mathbb{S}^{r} \rightarrow \mathrm{U}(n)$ is homotopic to its transposed or to its complex conjugate, respectively.

\begin{tabular}{|c|c|c|c|c|c|c|}
\hline$r \backslash n$ & 1 & 2 & 3 & 4 & 5 & 6 \\
\hline 1 & $\underset{\mathbb{Z}}{t}$ & $\mathbb{Z}$ & $\mathbb{Z}$ & $\mathbb{Z}$ & $\mathbb{Z}$ & $\mathbb{Z}$ \\
\hline 2 & 0 & 0 & 0 & 0 & 0 & 0 \\
\hline 3 & 0 & $\underset{c}{\mathbb{Z}}$ & $\mathbb{Z}$ & $\mathbb{Z}$ & $\mathbb{Z}$ & $\mathbb{Z}$ \\
\hline 4 & 0 & $\underset{t c}{\mathbb{Z}_{2}}$ & 0 & 0 & 0 & 0 \\
\hline 5 & 0 & $\mathbb{Z}_{t c}$ & $\underset{t}{\mathbb{Z}}$ & $\mathbb{Z}$ & $\mathbb{Z}$ & $\mathbb{Z}$ \\
\hline 6 & 0 & $\mathbb{Z}_{c}$ & $\mathbb{Z}_{c}$ & 0 & 0 & 0 \\
\hline 7 & 0 & $\underset{t c}{\mathbb{Z}_{2}}$ & 0 & $\underset{c}{\mathbb{Z}}$ & $\mathbb{Z}$ & $\mathbb{Z}$ \\
\hline 8 & 0 & $\underset{t c}{\mathbb{Z}_{2}}$ & $\mathbb{Z}_{t}$ & $\mathbb{Z}_{t} 24$ & 0 & 0 \\
\hline 9 & 0 & $\underset{c}{\mathbb{Z}_{3}}$ & $\underset{c}{\mathbb{Z}_{3}}$ & $\underset{t c}{\mathbb{Z}_{2}}$ & $\underset{t}{\mathbb{Z}}$ & $\mathbb{Z}$ \\
\hline 10 & 0 & $\underset{c}{\mathbb{Z}_{15}}$ & $\underset{c}{\mathbb{Z}_{30}}$ & $\mathbb{Z}_{120} \underset{c}{\oplus} \mathbb{Z}_{2}$ & $\underset{c}{\mathbb{Z}_{120}}$ & 0 \\
\hline
\end{tabular}

Table 1. Table of the first homotopy groups $\pi_{r} \mathrm{U}(n)$

\section{References}

[1] A. Borel, F. Hirzebruch, Characteristic classes and homogeneous spaces. II, Amer. J. Math. 81 (1959), 315-382.

[2] R. Bott, The space of loops on a Lie group, Michigan Math. J. 5 (1958), 35-61.

[3] R. Bott, The stable homotopy of the classical groups, Ann. of Math. 70 (1959), 313-337.

[4] R. Bott and J. Milnor, On the parallelizability of the spheres, Bull. Amer. Math. Soc. 64 (1958), 87-89. 
[5] L. M. Chaves and A. Rigas, Complex reflections and polynomial generators of homotopy groups, J. Lie Theory 6 (1996), 19-22.

[6] L. M. Chaves and A. Rigas, On a conjugate orbit of $G_{2}$, Math. J. Okayama Univ. 33 (1991), 155-161.

[7] B. Eckmann, Hurwitz-Radon matrices revisited: from effective solution of the Hurwitz matrix equations to Bott periodicity, in: The Hilton symposium 1993 (Montreal, PQ), 2335, CRM Proc. Lecture Notes, vol. 6, AMS, Providence, RI, 1994.

[8] B. Eckmann, Hurwitz-Radon matrices and periodicity modulo 8, Enseign. Math. 35 (1989), 77-91.

[9] B. Eckmann, Topology, algebra, analysis - relations and missing links, Notices Amer. Math. Soc. 46 (1999), 520-527.

[10] A. T. Fomenko, Variational principles of topology. Multidimensional minimal surface theory, Mathematics and its Applications (Soviet Series), 42. Kluwer, Dordrecht, 1990.

[11] W. Y. Hsiang and H. B. Lawson, Minimal submanifolds of low cohomogeneity, J. Differ. Geom. 5 (1971), 1-38.

[12] H. Kachi, Homotopy groups of homogeneous space $\mathrm{SU}(n) / \mathrm{SO}(n)$, J. Fac. Sci. Shinshu Univ. 13 (1978), 27-34.

[13] M. Kervaire, Non-parallelizability of the $n$-sphere for $n>7$, Proc. Natl. Acad. Sci. USA 44 (1958), 280-283.

[14] M. Kervaire, Some nonstable homotopy groups of Lie groups, Illinois J. Math. 4 (1960), 161-169.

[15] M. Kervaire, On the Pontryagin classes of certain $\mathrm{SO}(n)$-bundles over manifolds, Amer. J. Math. 80 (1958), 632-638.

[16] A. T. Lundell, A Bott map for non-stable homotopy of the unitary group, Topology 8 (1969) 209-217.

[17] A. T. Lundell, Concise tables of James numbers and some homotopy of classical Lie groups and associated homogeneous spaces, in: Algebraic topology (San Feliu de Guxols, 1990), 250-272, Lecture Notes in Math. 1509, Springer, Berlin, 1992.

[18] A. T. Lundell and Y. Tosa, Explicit construction of nontrivial elements for homotopy groups of classical Lie groups, J. Math. Phys. 31 (1990), 1494-1502.

[19] M. Mimura and H. Toda, Topology of Lie groups I, II, Translations of Mathematical Monographs, 91. AMS, Providence, 1991.

[20] G. F. Paechter, The groups $\pi_{r}\left(V_{n, m}\right)$. I, Quart. J. Math. Oxford Ser. (2) 7 (1956), 249-268.

[21] N. E. Steenrod, The Topology of Fibre Bundles, Princeton University Press, 1951.

T. Püttmann

Ruhr-Universität Bochum

Fakultät für Mathematik

D-44780 Bochum

Germany

e-mail: puttmann@math.ruhr-uni-bochum.de
A. Rigas

IMECC

UNICAMP, C.P. 6065

13083-970 Campinas, SP

Brazil

e-mail: rigas@ime.unicamp.br

(Received: September 23, 2002) 